\title{
$L$-functions of elliptic curves with complex multiplication, I
}

by

R. M. DAMEREELL (London)*

$\S 1$. Introduction. Let $\mathscr{C}$ be an elliptic curve

$$
y^{2}=4 x^{3}-a x-b
$$

where $a$ and $b$ are rationil constants. Birch and Swinnerton-Dyer have conjectured that the group of rational points on $\mathscr{C}$ may be related to the belaviour of the $L$-function $I_{\mathscr{E}}(s)$ of $\mathscr{C}$. For the definition of $L_{\mathscr{C}}(s)$ and an account of these conjectures, see Cassels [3], \&28. We follow Cassels in replacing the $s$ of Birch and Swinnerton-Dyer bs $s-\frac{1}{2}$, so that references to $s=1$ in the Birch-Swinnerton-Dyer notation are $s=\frac{1}{2}$ in the present notation.

We consider only the case when (1.1) has complex multiplication, so its ring $\boldsymbol{S}$ of endomorphisms over $\boldsymbol{C}$ is an order in some complex quadratic field $k=Q\left(\sqrt{-\bar{d}_{0}}\right)$, say. Then Deuring [4] has shown that

$$
L_{\mathscr{\varepsilon}}(s)=\prod_{\boldsymbol{p}}\left(1-\frac{\lambda(\boldsymbol{p})}{\mathcal{N}(\boldsymbol{p})^{s}}\right)^{-1}
$$

where $\boldsymbol{p}$ runs through the prime ideals of $\boldsymbol{S}$, except the finitely many for which $\mathscr{C}$ has bad reduction $\bmod \boldsymbol{p} . \mathscr{N} \boldsymbol{p}$ is the absolute norm of $\boldsymbol{p}$, that is the number of residues $\bmod \boldsymbol{p}$, and $\lambda$ is a Hecke Grössencharakter defined on the ideals of $\boldsymbol{S}$.

The product (1.2) converges when re $(s)>1$, but the function can be analytically continued over the whole complex plane. When $\boldsymbol{S}=\boldsymbol{Z}\left[1^{\prime}-1\right]$, Birch and Swinnerton-Dyer [1] showed how to construct a number $a$ such that

$$
L_{\mathscr{C}}\left(\frac{1}{2}\right) \times a=\tau(\mathscr{C}), \quad \text { say }
$$

* Most of this worl was done while the author was a researeh student at the University of Cambridge. 
is a rational integer. This result was useful, first because it is a considerable help in numerical computation, and second because it suggested the most reasonable form for the Birch-Swinnerton-Dyer conjectures.

More generally we may associate with $\mathscr{C}$ the functions

$$
I_{\mathscr{G}}^{[n]}(s)=\prod_{\boldsymbol{p}}\left(1-\frac{\lambda^{i n}(\boldsymbol{p})}{\mathscr{N}(\boldsymbol{p})^{s}}\right)^{-1}
$$

for any integer $n$, the case $n=1$ being (1.2). It is suspected that these funetions might give further information about rational points on $\mathscr{C}$, but no detailed conjectures have yet been macte.

In this series of papers we find for each odd $n$ a number $\alpha$ (depending on $\mathscr{C}$ and $n$, such that

$$
L_{\mathscr{G}}^{[n]}\left(\frac{1}{2}\right) \times a
$$

is a rational integer. The same proof applies to each of the 13 complex quadratic rings $\boldsymbol{S}$ with class number one. In this first paper it will be proved only that certain numbers are algebraic. Rationality and integrity will be proved later.

I would like to thank Professor J. W. S. Cassels and Mr. F. P. F. Swinnerton-Dyer for their most valuable assistance. Also I am grateful to Mr. Swinnerton-Dyer for showing me his manuscript [9] dealing with the case $n=3$, and for suggesting the general problem to me.

\$2. Statement of Theorem 1. We adopt the following notation. If $U$ is a ring, $U^{+}$shall denote the additive group of $U$, and $U^{*}$ the multiplicative group of units. Let $a_{0}$ be a square-free integer and $k_{0}$ the field $Q\left(\sqrt{-d_{0}}\right)$, with discriminant $d$ and ring of integers $\boldsymbol{R} . \boldsymbol{R}^{+}$is generated by 1 and $\sigma$ (say), where

$$
\begin{aligned}
& \text { if } d_{0} \equiv 3 \bmod 4, \quad \text { then } d=-d_{0} \text { and } \sigma=\frac{1}{2}+\frac{1}{2} \sqrt{-d_{0}}, \\
& \text { otherwise, } \\
& \qquad d=-4 d_{0} \text { and } \sigma=\sqrt{-d_{0}} .
\end{aligned}
$$

In either ease, $\operatorname{im}(\sigma)=\frac{1}{3} \mathrm{Y} / \dot{a} \mid$.

Let $\boldsymbol{S}$ be the subring $\boldsymbol{S}=\boldsymbol{Z}+f \boldsymbol{R}$, for some integer $\boldsymbol{f}$. An ideal $\boldsymbol{a}$ of $\boldsymbol{S}$ will be called proper if $\boldsymbol{a}$ is not a $\boldsymbol{T}$-module for any ring $\boldsymbol{T} \supset \boldsymbol{S}$. The proper ideals are the projective modules of rank one $\subseteq \boldsymbol{S}$. The proper fractional ideals are those finitely generated subgroups of $k^{+}$which are $\mathbf{S}$-modules but not $\boldsymbol{T}$-modules. They form a multiplicative group generated by the proper jorime ideals. The subgroup of principal ideals has finite index $h$, say. We can therefore construct a system ( $\mathfrak{B}$, say) of ideal numbers for the proper ideals of $\boldsymbol{S}$ in the same way as we form ideal numbers for the ideals of $\boldsymbol{R}$, See [8] for the method.
Let $\boldsymbol{m}$ be an ideal of $\boldsymbol{S}, \gamma_{1}$ a character on $(\boldsymbol{S} / \boldsymbol{m})^{*}$, and $\chi$ any extension of $\chi_{1}$ to $\mathfrak{B}$. A Hecke Grössencharazter is a function

$$
\lambda(\boldsymbol{a})=\left(\frac{\overline{\hat{\boldsymbol{a}}}}{|\hat{\boldsymbol{a}}|}\right)^{n} \chi(\hat{\boldsymbol{a}})
$$

defined on ideals $\boldsymbol{a}$ of $\boldsymbol{S}$, prime to $\boldsymbol{m}$. Here $\hat{\boldsymbol{a}}$ is an ideal number representing $\boldsymbol{a}$, and $\chi$ must be such that $\lambda(\boldsymbol{a})$ does not depend on the choice of $\boldsymbol{a}$. A Hecke zeta function with Grössencharakter is a function defined by the series

$$
\zeta(s, \lambda)=\sum \frac{\lambda(\boldsymbol{a})}{\mathscr{N}(\boldsymbol{a})^{s}},
$$

the sum running over proper ideals of $\boldsymbol{S}$ prime to $\boldsymbol{m}$

We use the standard notation for elliptic functions, as in Fricke [5] $\Omega$ shall denote a typical element, of a period lattice $\Gamma$. Lattice functions such as the Weierstrass elliptic function will be denoted by $\wp(z, T)$, etc. the argument $I$ being inserted only when necessary. We shall be considering series summed over: $T$, and the following lemma justifies the use of these series. The proof is left to the reader, but see Fricke [50], page 256.

Lewmi 2.1. Let $f(z)$ be a function such that $f(z) z^{-u}$ is bounded for some $u>2$. Then the series

$$
\sum_{\Omega \neq 0} f(z+\Omega)
$$

converges absolutely in the domain $\left(\boldsymbol{E}\right.$, say) of the $\left(z, \omega_{1}, \omega_{2}\right)$-space defined by these conditions:

(1) $\omega_{1} \neq 0, \omega_{2} \neq 0, \omega_{1} / \omega_{2}$ not real,

(2) $\approx \neq m_{1} \omega_{1}+m_{2} \omega_{2}$ for any $m_{1}, m_{2}$ in $\boldsymbol{Z}$.

The convergence is everywhere-locally uniform.

For any $x, \mathbf{S} x$ is a period lattice. Since $g_{2}(\mathbf{S} x)$ is homogeneous of degree $-t$ in $x, x$ can be chosen to make $g_{2}(\boldsymbol{S} ; x)$ an algebraic number. Then $g_{\mathrm{a}}(\boldsymbol{S} x)$ will also be algebraic, because $j(\boldsymbol{S} x)$ is algebraic (Hasse, [6], Satiz 8).

Some of the functions to be defined presently are many valued. In each case this happens because $z^{s}$ is a many valued function of $z$ and $s$. We assume from now on that a value of $\log (z)$ has been chosen for every $\approx(\neq 0)$, subject to the following

BRANCH CONVENTION. Whenever $z$ and $\bar{z}$ appear in the same formula, then $\log (\bar{z})$ is to be interpreted as $\log (z)$.

With this convention, everything defined in this paper is single valued, independently of the choice of $\log (z)$, but $\approx=\bar{z}$ does not imply 
$\log (z)=\log (\bar{z})$. In particular,

$$
z^{s} \bar{z}^{\varepsilon}=\exp (s(\log (z)+\log (\bar{z})))
$$

is single ralued. Also if $u+v$ is in $\boldsymbol{Z}$, then

\section{is single valued.}

$$
z^{u-s} / \bar{z}^{y+s}=(z /|z|)^{u+v}(z \bar{z})^{-s}
$$

This paper is devoted to the proof of

THEOREM 1. Let $\zeta(s, \lambda)$ be a Hecke zeta function defined by the series $(2.2)$, let $s_{0}$ be a value of $s$ such that $\frac{1}{2} n-s_{0} \in \boldsymbol{Z}$ and $0<s_{0} \leqslant \frac{1}{2} n$, and let $x$ be chosen to make $g_{2}(\mathbf{S} x)$ and $g_{3}(\boldsymbol{S} x)$ atgebraic numbers. Then $\zeta(s, \lambda)$ is regular at $s=s_{0}$ and the number.

is algebraic.

$$
\zeta\left(s_{0}, \lambda\right) \pi^{n / 2-s_{0}} / x^{n}=\Theta, \text { say }
$$

By substituting $-n$ for $n$ and $\overline{\boldsymbol{a}}$ for $\boldsymbol{a}$ in $(2.2)$ if need be, we may assume that $n \geqslant 0$. But if $n=0$, the theorem is without content, because $0<\delta_{0} \leqslant \frac{1}{2} n$ is impossible. The basic idea of the proof is that $\zeta(s, \lambda)$ will be expressed in terms of certain doubly periodic functions. We proceed to construct them.

§3. The functions $K_{j}^{i}(z, T)$. We adopt the convention that in series summed over $\Omega$ any term in angle brackets is to be omitted when $\Omega=0$. Let

$$
\zeta(z, T)=\sum_{D \in I^{\prime}}\left\{\frac{1}{z+\Omega}-\left\langle\frac{1}{\Omega}-\frac{z}{\Omega^{2}}\right\rangle\right\}
$$

be the Weierstrass zeta function. Then

$$
\text { (3.2) } \quad \zeta(z+\Omega)=\zeta(z)+\eta
$$
where $\eta=m_{1} \eta_{1}+m_{2} \eta_{2}$ if $\Omega=m_{1} \omega_{1}+m_{2} \omega_{2} . \eta_{1}$ and $\eta_{2}$ are constants
which satisfy

$$
\omega_{1} \eta_{2}-\omega_{2} \eta_{1}=2 \pi i
$$

(see Fricke [5], page 160).

Defrintion. For integers $i, j$ with $0 \leqslant i<j$ define

(3.4) $K_{j}^{i}(z, T)$

$$
=\left\{\begin{array}{ll}
\sum_{\text {SeT }}\left\{\frac{\zeta^{i}(z+\Omega)}{(z+\Omega)^{j}}\right\} & \text { if } \quad j>i+2, \\
\sum_{\operatorname{QeT}}\left\{\frac{\zeta^{i}(z+\Omega)}{(z+\Omega)^{i}}-\left\langle\frac{\eta^{i}}{\Omega^{i}}\right)\right\} & \text { if } j=i+2, \\
\sum_{\Omega \in T}\left\{\frac{\zeta^{i}(z+\Omega)}{(z+\Omega)^{j}}-\left\langle\frac{\eta^{i}}{\Omega^{j}}\left(1+\frac{i \zeta(z)}{\eta}-\frac{j z}{\Omega}\right)\right\rangle\right\} & \text { if } j=i+1 .
\end{array},\right.
$$

LEMDA 3.1. $K_{j}^{i}$ is a well defined regular function of $\omega_{1}, \omega_{2}$ and $\approx i n$ the domain $\boldsymbol{E}$ of Lemma 2.1. The series may be differentiated term by term. For fixed $\Gamma, K_{j}^{i}$ has poles of order $i+j$ at the lattice points.

Proof. Each term of each series in (3.4) is a regtlar function of $\omega_{1}, \omega_{2}$ and $z$ in $\boldsymbol{E}$. Moreover as $|\Omega| \rightarrow \infty$,

$$
\zeta(z+\Omega)=\zeta(z)+\eta=\eta+O(1) \quad \text { and } \quad \eta=O\left(m_{1}, m_{2}\right)=O(\Omega) .
$$

It can now be checked that the three summands in (3.4) are $O\left(\Omega^{-3}\right)$ as $|\Omega| \rightarrow \infty$. By Lemma, 2.1 the series (3.4) are each locally uniformly convergent in $E$, so $K_{j}^{i}$ is regular there.

Now fix $T$ and consider the pole of $K_{j}^{i}$ at a lattice point $z=\Omega$. The term $\zeta^{i}(z-\Omega) /(z-\Omega)^{i}$ has a pole of order $i+j$ and every other term has a pole of order $i$. So $K_{j}^{i}$ has a pole of order $i+j$. Q.E.D.

Let

$$
D=D_{\eta}+\zeta(z) \frac{\partial}{\partial z}
$$

where $D_{\eta}=\eta_{1} \frac{\partial}{\partial \omega_{1}}+\eta_{2} \frac{\partial}{\partial \omega_{2}}$ is as in Fricke [5], page 316. From Fricke's formulae (pages $316,322,325)$ we deduce

$$
\left\{\begin{array}{l}
D(\Omega)=D_{\eta}\left(m_{1} \omega_{1}+m_{2} \omega_{2}\right)=m_{1} \eta_{1}+m_{2} \eta_{2}=\eta \\
D(\eta)=D_{\eta}\left(m_{1} \eta_{1}+m_{2} \eta_{2}\right)=-\frac{1}{12} g_{2}\left(m_{1} \omega_{1}+m_{2} \omega_{2}\right)=-\frac{1}{12} g_{2} \Omega \\
D(z)=\zeta(z) \\
D(\zeta(z))=\frac{1}{2} \wp^{\prime}(z)-\frac{1}{12} g_{2} z \\
D(z+\Omega)=\zeta(z)+\eta=\zeta(z+\Omega), \\
D(\zeta(z+\Omega))=\frac{1}{2} \wp^{\prime}(z)-\frac{1}{12} g_{2}(z+\Omega) .
\end{array}\right.
$$

LeinMa 3.2 .

(3.6) $D\left(K_{j}^{i}(z, T)\right)=\frac{1}{2} i \wp^{\prime}(z) K_{j}^{i-1}(z, \Gamma)-\frac{1}{12} i g_{2} K_{j-1}^{i-1}(z, \Gamma)-j K_{j+1}^{i+1}(z, \Gamma)$.

Proof. First suppose $j>i+2$. Differentiate the series for $K_{i}^{i}$ term by term:

$$
\begin{aligned}
& D\left(K_{j}^{i}(z, \Gamma)\right)=\sum_{\Omega \in T} D\left(\frac{\zeta^{i}(z+\Omega)}{(z+\Omega)^{i}}\right) \\
& =\sum_{\Omega \in \Gamma}\left[\frac{1}{2} i \wp^{\prime}(z)\left\{\frac{\zeta^{i-1}(z+\Omega)}{(z+\Omega)^{j}}\right\}-\frac{1}{12} i g_{2}\left\{\frac{\zeta^{i-1}(z+\Omega)}{(z+\Omega)^{j-1}}\right\}-j\left\{\frac{\zeta^{i+1}(z+\Omega)}{(z+\Omega)^{j+1}}\right\}\right]
\end{aligned}
$$

by equations (3.5). Each of the terms in curly brackets is $O\left(\Omega^{-3}\right)$ as $|\Omega| \rightarrow \infty$, so they can be summed separately over $\Omega$. This gives equation (3.6), and proves the Lemma when $j>i+2$. If $j=i+2$ or $j=i+1$, Acta Arithmetica XVII.3 
the proof is similar but more complicated due to the presence of angle bracket terms. Details are left to the reader.

Defrnmon. A standard function is a polynomial in $\wp(z), \frac{1}{2} \wp^{\prime}(z)$, $\frac{1}{12} g_{2}$, and $\frac{1}{4} g_{3}$, with coefficients in $\boldsymbol{Z}$. A standard constant is a standard function that depends only on $g_{2}$ and $g_{3}$.

From formulae given by Fricke ([5], pages 317, 322) we deduce:

$$
\left\{\begin{array}{l}
D\left(\frac{1}{12} g_{2}\right)=D_{\eta}\left(\frac{1}{12} g_{2}\right)=-\frac{1}{2} g_{3}, \\
D\left(\frac{1}{4} g_{3}\right)=D_{\eta}\left(\frac{1}{\frac{1}{2}} g_{3}\right)=-\frac{1}{12} g_{2}^{2}, \\
D(\wp(z))=\left(D_{\eta}+\zeta(z) \frac{\partial}{\partial z}\right)(\wp(z))=-2 \wp^{2}(z)-\frac{1}{3} g_{2}, \\
D\left(\frac{1}{2} \wp^{\prime}(z)\right)=\frac{1}{2} \frac{\partial}{\partial z}\left(D_{\eta}(\wp(z))\right)+\frac{1}{2} \zeta(z) \wp^{\prime \prime}(z)=-\frac{3}{2} \wp(z) \wp^{\prime}(z)
\end{array}\right.
$$

Hence $D(V)$ is a standard function if $V$ is. We now have

LENMA 3.3 .

$$
(j-1) ! K_{j}^{i}(z)=T_{j}^{i}(z)+z A_{j}^{i}+\zeta(z) B_{j}^{i},
$$

where $T_{j}^{i}$ is a standard function, $A_{j}^{i}$ and $B_{j}^{i}$ are standard constants, and $A_{j}^{i}=B_{j}^{i}=0$ if $j \geqslant i+2$.

Proof. First suppose $i=0$. Consider the series (3.4) that define $K_{j}^{i}$. If $j=1$, then

$$
K_{1}^{0}(z)=\sum\left\{\frac{1}{z+\Omega}-\left\langle\frac{1}{\Omega}-\frac{z}{\Omega^{2}}\right\rangle\right\}_{-}=\zeta(z)
$$

by (3.1). So (3.8) holds with $x_{1}^{0}=0, A_{1}^{0}=0, B_{1}^{0}=1$. If $j=2$, then

$$
K_{2}^{0}(z)=\sum\left\{\frac{1}{(z+\Omega)^{2}}-\left\langle\frac{1}{\Omega^{2}}\right\rangle\right\}=\wp(z)
$$

So (3.8) holds with $A_{2}^{0}=B_{2}^{0}=0, T_{2}^{0}=\wp(z)$, a standard function. For $j \geqslant 3$;

$$
(j-1) ! K_{j}^{0}(z)=\sum \frac{(j-1) !}{(z+\Omega)^{j}}=(-1)^{j} \frac{\partial^{j-2}}{\partial z^{j-2}}(\wp(z)) .
$$

To prove the Lemma when $i=0$ and $j \geqslant 3$, it is enough to show that the derivatives of $\wp(z)$ are standard functions. This is proved by differentiation, using the formulae

(3.10)

and

$$
\left(\frac{1}{2} \wp \rho^{\prime}(z)\right)^{2}=\wp^{3}(z)-\frac{1}{4} g_{2} \wp(z)-\frac{1}{4} g_{3}
$$

$$
\wp^{\prime \prime}(z)=6 \wp^{2}(z)-\frac{1}{2} g_{2}
$$
(3.6),

Now suppose the Lemma proved for all integers $i^{\prime} \leqslant i$. By equation

(3.12) $j ! K_{j+1}^{i+1}(z)$

$$
=i\left(\frac{1}{2} \wp^{\prime}(z)\right)\left[(j-1) ! K_{j}^{i-1}(z)\right]-i\left(\frac{1}{12} g_{2}\right)\left[(j-1) ! K_{j-1}^{i-1}(z)\right]-D\left[(j-1) ! K_{j}^{i}(z)\right] .
$$

If $j \geqslant i+2$, then by the inductive hypothesis the terms on the right hand side of (3.12) are standard functions, hence so is $j ! K_{j+1}^{i+1}$. This establishes the induction from $i$ to $i+1$. If $j=i+1$, the inductive hypothesis is

$$
(j-1) ! K_{j}^{i}(z)=T_{j}^{i}(z)+z A_{j}^{i}+\zeta(z) B_{j}^{i} .
$$

Applying $D$ gives

$$
\begin{aligned}
D\left((j-1) ! K_{j}^{i}(z)\right) & =D\left(T_{j}^{i}\right)+z D\left(A_{j}^{i}\right)+\zeta(z) D\left(B_{j}^{i}\right)+A_{j}^{i} D(z)+B_{j}^{i} D(\zeta(z)) \\
& =D\left(T_{j}^{i}\right)+\frac{1}{2} \wp^{\prime}(z) B_{j}^{i}+z\left[D\left(A_{j}^{i}\right)-\frac{1}{12} g_{2} B_{j}^{i}\right]+\zeta(z)\left[D\left(B_{j}^{i}\right)+A_{j}^{i}\right] .
\end{aligned}
$$

This has the form of (3.8) because the terms in square brackets are standard constants. Then all the terms on the right hand side of (3.12) have the form of (3.8). This establishes the induction when $j=i+1$. Q.F.D.

\section{\$ 4. Periodic non-analytic functions.}

Defrnicton. A function $f(z)$ shall be called quasi-periodic if there are constants $a_{1}, a_{2}$ such that

$$
\begin{aligned}
& f\left(z+\omega_{1}\right)=f(z)+a_{1}, \\
& f\left(z+\omega_{2}\right)=f(z)+a_{2}
\end{aligned}
$$

for all $z$.

LEMMA 4.1. If $f(z)$ is quasi-periodic, there is precisely one pair of constants $\alpha, \beta$ such that

$$
f(z)+\alpha z+\beta \bar{z}
$$

is periodic.

Proof. (4.2) will be periodic if and only if

$$
\begin{aligned}
& a_{1}+\alpha \omega_{1}+\beta \bar{\omega}_{1}=0, \\
& a_{2}+\alpha \omega_{2}+\beta \bar{\omega}_{2}=0 .
\end{aligned}
$$

The determinant of this pair of equations is

$$
\left(\omega_{1} \bar{\omega}_{2}-\omega_{2} \bar{\omega}_{1}\right)=\omega_{2} \bar{\omega}_{2}(\omega-\bar{\omega})
$$

where $\omega=\omega_{1} / \omega_{2}$. So equations (4.3) can be uniquely solved for $\alpha, \beta$ since $\omega_{2} \neq 0$ and $\omega$ is not real. 
DEFINITION.

$$
h(z)=\zeta(z)+\varphi z+\psi \bar{z}
$$

where $\zeta(z)$ is the Weierstrass zeta function and $p$ and $\psi$ are the unique pair of constants that make $h(z)$ doubly periodic.

LEMnI 4.2. Let $\boldsymbol{b}$ be an ideal in $\mathbf{S}$ and $x$ any number. Then

$$
\pi / x \bar{x}=\psi(\boldsymbol{b} x)\left(-\frac{1}{2} f \mathscr{N}(\boldsymbol{b}) \sqrt{|\bar{d}|}\right) .
$$

Proof. Let $A(T)$ denote the area of a parallelogram of $I$. Put $\alpha=q$, $\beta=\psi, a_{i}=\eta_{i}$ in equations (4.3) and solve for $\psi$. This gives

$$
\psi(\Gamma)=\frac{\omega_{2} \eta_{1}-\omega_{1} \eta_{2}}{\omega_{1} \bar{\omega}_{2}-\omega_{2} \bar{\omega}_{1}}=\frac{-2 i \pi}{2 i A(\Gamma)}=\frac{-\pi}{A(\Gamma)}
$$

by equation (3.3) and simple trigonometry. By the results quoted in $\$ 2$, $A(\boldsymbol{R})=\frac{1}{2} \sqrt{|d|}$. Also $\boldsymbol{b}$ has index $\mathscr{N}(\boldsymbol{b})$ in $\boldsymbol{S}$ which has index $f$ in $\boldsymbol{R}$, so

$$
-\psi(\boldsymbol{b} x)=\frac{\pi}{A(\boldsymbol{b} x)}=\frac{\pi}{x \bar{x} A(\boldsymbol{b})}=\frac{\pi}{x \bar{x}} \cdot \frac{1}{\frac{1}{2} f \mathcal{N}(\boldsymbol{b}) \sqrt{|d|}}
$$

hence $(4.5)$.

Defrimiron. For integer $m$, define

$$
H_{m}(z)=\frac{1}{2 m}\left\{\frac{\wp^{\prime \prime}(z)}{\wp^{\prime}(z)}+\sum_{r=2}^{m-2} \frac{\wp^{\prime}(r z)-\wp^{\prime}(z)}{\wp(r z)-\wp(z)}\right\} .
$$

LENMA 4.3. $H_{m}(z)$ is equal in value to $h(z)$ whenever $m z$ is in $\Gamma$ but $\approx$ is not in $\Gamma$.

Proof. This lemma is due to Swinnerton-Dyer, [9]. Consider the addition formula

$$
\zeta(u+v)=\zeta(u)+\zeta(v)-\frac{1}{2} \frac{\wp^{\prime}(u)-\wp^{\prime}(v)}{\wp(u)-\wp(v)}
$$

(Fricke [5], page 202). In the limit as $v \rightarrow u$, this becomes

$$
\zeta(2 u)=2 \zeta(u)-\frac{1}{2} \frac{\wp^{\prime \prime}(u)}{\wp^{\prime}(u)} .
$$

Apply (4.7) repeatedly and (4.8) once to (4.6). This gives

$$
\begin{aligned}
H_{m}(z) & =\frac{1}{m}\{(m-1) \zeta(z)-\zeta([m-1] z)\} \\
& =\frac{1}{m}\{(m-1) h(z)-h([m-1] z)\}
\end{aligned}
$$

because the linear terms in $z$ and $\bar{z}$ cancel.
Suppose now that $m z \in \Gamma$ but $z \notin \Gamma$. Then $(m-1) z \notin \Gamma$, so $(4.9)$ is finite. Since $h(z)$ is an odd and periodic function of $z$,

$m H_{m}(z)=(m-1) h(z)-h([m-1] z)=(m-1) h(z)-h(-z)=m h(z)$, Q.E.D.

Defintaron. A substandard function is a polynomial in $h(z), \wp(z)$, $\wp^{\prime}(z), g_{2}, g_{3}$ and $\varphi$, with rational coefficients.

Coroliary 4.1 (to Lemma 3.3). $K_{j}^{i}(z)$ is periodic if $j \geqslant i+2$ and quasi-periodio if $j=i+1$. If $\alpha, \beta$ be chosen to make $K_{j}^{i}(z)+\alpha z+\beta \bar{z}$ periodio (so $\alpha=\beta=0$ if $j \geqslant i+2)$, then

$$
(j-1) !\left(K_{j}^{i}(z)+\alpha z+\beta \bar{z}\right)=T_{j}^{i}(z)+B_{j}^{i} h(z),
$$

where $B_{j}^{i}=0$ if $j \geqslant i+2$. This is a substandard function.

Proof. By Lemma 3.3,

$$
(j-1) ! K_{j}^{i}(z)=T_{j}^{i}(z)+A_{j}^{i} z+B_{j}^{i} \zeta(z) .
$$

This is quasi-periodic if $j=i+1$ and periodic if $j \geqslant i+2$ because then $A_{j}^{i}=B_{j}^{i}=0$. So (4.10) holds if $j \geqslant i+2$. If $j=i+1$, then by adding multiples of $z$ and $\bar{z}$ we may replace $\zeta(z)$ by $h(z)$ and cancel the term in $z$. By Lemma 4.1, these multiples of $z$ and $\vec{z}$ are unique. This gives (4.10). $T_{j}^{i}$ is a standard function and $B_{j}^{i}$ a standard constant, so (4.10) is substandard.

§ 5. The function $F_{n}(z, s, \Gamma)$.

DEFINITION. Let $u, v, z, s$, be complex numbers such that $u+v \in \boldsymbol{Z}$, and $n$ an integer. Define

$$
\begin{gathered}
F_{n}(z, s, \Gamma)=\sum_{\Omega} \frac{(\bar{z}+\bar{\Omega})^{n / 2-s}}{(z+\Omega)^{n / 2+s}} \\
G_{v}^{u}(z, s, \Gamma)=\sum_{\Omega}\left\{\frac{(\bar{z}+\bar{\Omega})^{u-s}}{(z+\Omega)^{v+s}}-\left\langle\frac{\bar{\Omega}^{u-s}}{\Omega^{v+s}}\right\rangle\right\} \\
W_{v}^{u}(s, I)=\sum_{\Omega \neq 0} \frac{\bar{\Omega}^{u-s}}{\Omega^{v+s}}
\end{gathered}
$$

By the branch convention each term of each series is single valued. By Lemma 2.1, the series converge in the respective domains

and

$$
\begin{aligned}
& \operatorname{re}(s)>1, \\
& \operatorname{re}(s)>\frac{1}{2}+\frac{1}{2}(u-v) \\
& \operatorname{re}(s)>1+\frac{1}{2}(u-v)
\end{aligned}
$$

and they represent regular functions there. 
The object of this section is to show that if $s_{0}$ is as in Theorem 1 , then $F_{n}\left(z, s_{0}, T\right)$ can be expressed in terms of a substandard function. This requires the analytic continuation of $F_{n}$ beyond $s=1$. To prove analytic continuation we must assume that $\Gamma$ admits complex multiplication.

LEMNA 5.1. Suppose that $T$ admits complex multiplication by the ring S. If. $u+v=0, W_{v}^{u}$ has at worst a simple pole at $s=1+\frac{1}{2}(u-v)$.' If $u+v \neq 0, W_{v}^{u}$ is regular there and its value is given by

$$
\text { (5.4) } \quad W_{v}^{u}\left(1+\frac{1}{2} u-\frac{1}{2} v\right)=\left[\tau \bar{\tau}\left(\{\tau /|\tau|\}^{u+v}-1\right)\right]^{-1} \sum_{\varrho} G_{v}^{u}\left(\varrho / \tau, 1+\frac{1}{2} u-\frac{1}{2} v\right)
$$

where $\tau$ is in $\mathbf{S}, \tau^{u+v}$ is not real and $Q$ runs through the non-zero residues of $\Gamma$ modulo $\tau T$.

Proof. Take any $\tau \neq 0$ in $S$. Every $\Omega \neq 0$ in $\Gamma$ can be uniquely expressed as

$$
\Omega=\tau \Omega^{\prime} \quad\left(\Omega^{\prime} \neq 0\right) \quad \text { or } \quad \Omega=\varrho+\tau \Omega^{\prime} . \quad(\varrho \neq 0) .
$$

So (5.3) gives

$$
W_{v}^{u}(s)=\sum_{\Omega \neq 0} \frac{(\bar{\tau} \bar{\Omega})^{u-s}}{(\tau \Omega)^{v+s}}+\sum_{\varrho \neq 0} \sum_{\Omega} \frac{(\varrho+\bar{\tau} \bar{\Omega})^{u-s}}{(\varrho+\tau \Omega)^{v+s}}
$$

Hence

$$
W_{v}^{u}(s)\left(\tau^{v+s} / \bar{\tau}^{u-s}-1\right)=\sum_{\varrho \neq 0} \sum_{\Omega} \frac{(\varrho / \bar{\tau}+\bar{\Omega})^{u-s}}{(\varrho / \tau+\Omega)^{v+s}}
$$

But there are $(\bar{\tau}-1)$ values of $\varrho$, so

$$
(\tau \bar{\tau}-1) W_{v}^{u}(s)=\sum_{e \neq 0} \sum_{\Omega \neq 0} \frac{\bar{\Omega}^{u-s}}{\Omega^{v+s}}
$$

By subtraction,

$$
W_{v}^{u}(s)\left[\tau^{v+s} / \bar{\tau}^{u-s}-\tau \bar{\tau}\right]=\sum_{\varrho \neq 0} G_{v}^{u}(\varrho / \tau, s) .
$$

Call the factor in square brackets $U(s)$. Since $G_{v}^{u}$ is regular in $r e(s)$ $>\frac{1}{2}+\frac{1}{2}(u-v), W_{v}^{u}$ is regular there except for poles at the points where $U(s)=0$. Now

$$
U(s)=\tau^{v+s} / \bar{\tau}^{u-s}-\tau \bar{\tau}=(\tau /|\tau|)^{u+v}(\tau \bar{\tau})^{s+(v-u) / 2}-\tau \bar{\tau}
$$

and

$$
U^{\prime}(s)=\log (\tau \bar{\tau})(U(s)+\tau \bar{\tau})
$$

Clearly the zeroes of $U(s)$ are simple, so the poles of $W_{v}^{u}$ are at worst simple. If $u+v \neq 0$, put $s=1+\frac{1}{2}(u-v)$ in (5.6). This gives

$$
U\left(1+\frac{1}{2} u-\frac{1}{2} v\right)=\tau \bar{\tau}\left(\{\tau /|\tau|\}^{u+v}-1\right) \neq 0
$$

provided that $\tau^{u+v}$ is not real. Hence $W_{v}^{u}$ is regular at $s=1+\frac{1}{2}(u-v)$, and substituting (5.7) into (5.5) gives (5.4).

LIEMMA 5.2. Let $s_{0}$ be such that $\frac{1}{2} n-s_{0} \epsilon \boldsymbol{Z}$ and $0<s_{0} \leqslant \frac{1}{2} n$. Put $p=\frac{1}{2} n-s_{0}$ and $q=\frac{1}{2} n+s_{0}$. Assume that $\Gamma$ admits complexs multiplication by S. Then $F_{n}(z, s, T)$ is regular at $s=s_{0}$, and

(5.8) $\psi^{p} F_{n}\left(z, s_{0}\right)-B=\sum_{t+u+v=p}\left[\frac{p !}{t ! u ! v !} \hbar(z)^{t}(-\varphi)^{u}(-1)^{v} K_{q-u}^{v}(z)\right]+C z+D \bar{z}$,

where $B=0$ unless $s_{0}=1$ and $C=D=0$ untess $s_{0}=\frac{1}{2}$. The right hand side of $(5.8)$ is a substandard function $\left(U(z)\right.$, say). If $s_{0}=1$, then

$$
B=\left[\tau \bar{\tau}\left(\{\tau /|\tau|\}^{n}-1\right)\right]^{-1} \sum_{\varrho \neq 0} U(\varrho / \tau)
$$

where $\tau$ is an element of $\mathbf{S}, \tau^{n}$ not real, and e runs through the non-zero residues of $\Gamma$ modulo $\tau \Gamma$.

Proof. First suppose $s_{0}>1$. Since

$$
h(z)=\zeta(z)+\varphi z+\psi \bar{z}
$$

is periodic, it follows that

$$
h(z)=\zeta(z+\Omega)+\varphi(z+\Omega)+\psi(\bar{z}+\bar{\Omega}) .
$$

Multiply (5.1) by $\psi^{p}$, substitute for $\psi(\bar{z}+\bar{\Omega})$ and expand the numerator by the Binomial Theorem. This gives

$$
\begin{aligned}
\psi^{p} F_{n}\left(z, s_{0}\right) & =\sum_{\Omega} \frac{\left(h(z)-\varphi(z+\Omega)-\left.\zeta(z+\Omega)\right|^{p}\right.}{(z+\Omega)^{q}} \\
& =\sum_{\Omega}\left[\sum_{t+u+v=p} \frac{p !}{t ! u ! v !} h(z)^{t}(-\varphi)^{u}(-1)^{v}\left\{\frac{\zeta^{v}(z+\Omega)}{(z+\Omega)^{q-u}}\right\}\right]
\end{aligned}
$$

As $|\Omega| \rightarrow \infty$, the term in curly brackets is $O\left(\Omega^{v-(q-u)}\right)$. But $v-(q-u)$ $=p-t-q=-t-2 s_{0}<-2$. So we may sum these terms over $\Omega$ before summing over $t, u, v$, by Lemma 2.1. This gives (5.8), with $B=C=D=0$. Next suppose $n$ is even, and $s_{0}=1$. When $\mathrm{re}(s)$ is large the series (5.1) can be rearranged as follows:

$$
\begin{aligned}
F_{n}(z, s) & =\sum_{\Omega}\left\{\frac{(\bar{z}+\bar{\Omega})^{n / 2-s}}{(z+\Omega)^{n / 2+s}}-\left\langle\frac{\bar{\Omega}^{n / 2-s}}{\Omega^{n / 2-s}}\right\}\right\}+\sum_{\Omega \neq 0} \frac{\bar{\Omega}^{n / 2-s}}{\Omega^{n / 2+s}} \\
& =G_{n / 2}^{n / 2}(z, s)+W_{n / 2}^{n / 2}(s)
\end{aligned}
$$


Both terms are regular at $s=1$, by Lemma 5.1. It can now be proved. that when $s=1$,

$$
\psi^{p} G_{n j a}^{n j+2}(z, 1)=\sum_{t+u+v=p} \frac{p !}{t ! u ! v !} h(z)^{t}(-\varphi)^{u}(-1)^{v} K_{q-u}^{v}(z) .
$$

The method is the same as before, but the details are more complicated because the sum over $Q$ contains angle-bracket terms. Combining (5.10) and (5.11) gives (5.8), with $O=D=0$.

Now suppose $n$ is odd, and $s_{0}=\frac{1}{2}$. When re $(s)$ is large the series (5.1) may be rearranged as follows:

(5.12) $\quad F_{n}(z, s)$

$$
\begin{aligned}
= & \sum_{\Omega}\left\{\frac{(\bar{z}+\bar{\Omega})^{n / 2-s}}{(z+\Omega)^{n / 2+s}}-\left\langle\frac{\overline{\Omega^{n / 2-s}}}{\Omega^{n / 2+s}}\left(1-\left(\frac{1}{2} n+s\right) \frac{z}{\Omega}+\left(\frac{1}{2} n-s\right)+\frac{\bar{z}}{\bar{Q}}\right)\right\rangle\right\}+ \\
& +\sum_{\Omega \neq 0} \frac{\bar{\Omega}^{n / 2-s}}{\Omega^{n / 2+\delta}}-\left(\frac{1}{2} n+s\right) z \sum_{\Omega \neq 0} \frac{\bar{\Omega}^{n / 2-s}}{\Omega^{n / 2+s+1-1}}+\left(\frac{1}{2} n-s\right) \bar{z} \sum_{s \neq \neq 0} \frac{\bar{\Omega}^{n / 2-s-1}}{\Omega^{n / 2+s}} .
\end{aligned}
$$

The first series is regular in re $(s)>0$, by Lemma 2.1. Since $n$ is odd the second series ranishes because the terms in $1 \Omega$ cancel. The third and fourth series are $W_{n+2+1}^{n / 2}$ and $W_{n / 2}^{n / 2-1}$; these are regular at $s=\frac{1}{2}$ by Lemma 5.1, except that $W_{n / 2}^{n / 2-1}$ may have a simple pole at $s=\frac{1}{2}$ if $n=1$. But then $\left(\frac{1}{2} n-s\right)$ has a simple zero so the product is regular. So $F_{n}$ is regular. at $s=\frac{1}{2}$. By the same method as before, we have

$$
\psi^{p} F_{n}\left(z, \frac{1}{2}\right)=\sum_{t+u \div v=p} \frac{p !}{t ! u ! v !} h(z)^{t}(-\varphi)^{u}(-1)^{v} K_{q-u}^{v}(z)+C z+D z
$$

This is (o.8), with $B=0$. Observe that

$$
C=-\left(\frac{1}{2} n+\frac{1}{2}\right) \psi^{p} W_{n / 2+1}^{n / 2}\left(\frac{1}{2}\right)
$$

So (5.8) holds for all values of $s_{0}$.

Next consider the functions $K_{q-u}^{v} \cdot(q-u)-v=t+2 s_{0}$, so by Corollary 4.1 the $K_{q-u}^{v}$ are periodic unless $s_{0}=\frac{1}{2}$ and $t=0$. Fence the nonpexiodic $K$ 's occur only with constant coefficients in (5.8), and by adding multiples of $z$ and $\bar{z}$ we can replace each of them by the periodic function $K_{q-z}^{u}(z)+\alpha z+\beta \bar{z}$, which is a substandard function by Corollary 4.1 . Then (5.8) becomes.

$$
\begin{aligned}
& \psi^{n} F_{n}\left(z, s_{0}\right)-B \\
= & \sum_{t+u+v=p} \frac{p !}{t ! u ! v !} h(z)^{t}(-\varphi)^{u}(-1)^{v}\left(K_{q-u}^{v}(z)+\alpha z+\beta z\right)+C_{1} z+D_{1} \bar{z}
\end{aligned}
$$

But the series (气.I) is a periodic function of $z$ and so $F_{n}$ is periodic. So the constants $O_{1}$ and $D_{1}$ must vanish by the uniqueness part of Lemma 4.1. Fence (5.15) is a substandard function $(U(z)$, say).

Finally, consider $B$ when $s_{0}=1$. By comparing (5.15) and (5.10) we seo that $U(z)=\psi^{p} G_{n / 2}^{n / 2}(z, 1)$ and that

$$
\begin{aligned}
B & =\psi^{p} W_{n / 2}^{n / 2}(1)=\psi^{p}\left[\tau \bar{\tau}\left(\{\tau /|\tau|\}^{n}-1\right)\right]^{-1} \sum_{\varrho \neq 0} G_{n / 2}^{n j \mid}(\varrho / \tau, 1) \\
& =\left[\tau \bar{\tau}\left(\{\tau /|\tau|\}^{n}-1\right)\right]^{-1} \sum_{\varrho \neq 0} U(\varrho / \tau),
\end{aligned}
$$

by Lemma 5.1. This completes the proof of Leroma 5.2.

\section{§ 6. Proof of Theorem 1.}

Lemma 6.1. Let $x$ and $s_{0}$ be as in Theorem 1. (That is $g_{2}(\mathbf{S} x)$ and $g_{3}(\mathbf{S} x)$ are algebraic and $\frac{1}{2} n-s_{0} \in \boldsymbol{Z}$ and $0<s_{0} \leqslant \frac{1}{2} n$.) Let $\Gamma=\boldsymbol{b} x$ where $\boldsymbol{b}$ is an ideal of $\mathbf{S}$, and let $z$ have finite order (m, say) modulo $T$.

Then $\psi^{n / 2-s_{0}} F_{n}\left(z, s_{0}, \boldsymbol{b} x\right)$ is an algebraic number.

Proof. Let $\Delta$ be a sublattice of $\Gamma$. By comparing $\Omega$-series, we see that

$$
g_{2}(\Gamma)=g_{2}(\Delta)+10 \sum_{e} \wp^{\prime \prime}(\varrho, \Delta)
$$

where $\varrho$ runs through the non-zero residues of $T$ modulo $\Delta$. Choose $a(\neq 0)$ in $\boldsymbol{b} \cap \boldsymbol{Z}$ and put $\Gamma=\boldsymbol{b} x$ and $\Delta=\boldsymbol{S} a x$. Each $\varrho$ has order $\boldsymbol{a}$ modulo $\Delta$ and $g_{2}(\boldsymbol{S} x)$ and $g_{3}(\boldsymbol{S} x)$ are algebraic. So the $\wp^{\prime \prime}(\varrho, \boldsymbol{S} a x)$ are algebraic, hence so is $g_{2}(\boldsymbol{b} x)$. Similarly, $g_{3}(\boldsymbol{b} x)$ is algebraic. Since $z$ has order $m$ modulo $\boldsymbol{b} x$, it follows by standard theory that $\wp(z, \boldsymbol{b} x)$ and $\wp^{\prime}(z, \boldsymbol{b} x)$ are algebraic, and so is

$$
h(z)=H_{m}(z)=\frac{1}{2 m}\left\{\frac{\wp^{\prime \prime}(z)}{\wp^{\prime}(z)}+\sum_{r=2}^{m-2} \frac{\wp^{\prime}(r z)-\wp^{\prime}(z)}{\wp(r z)-\wp(z)}\right\}
$$

by Lemma 4.3 .

Now consider $q$. Put $n=1$ in equation (5.8). Since $p=\frac{1}{2} n-\frac{1}{2}=0$ and $q=\frac{1}{2} n+\frac{1}{2}=1,(5.8)$ reduces to

$$
F_{1}\left(z, \frac{1}{2}, T\right)=K_{1}^{0}(z)+C z+D \bar{z}
$$

But $K_{1}^{0}(z)=\zeta(z)$ by equation (3.9). Since $F_{1}$ is periodic it follows from (4.4) and Lemma 4.1 that $O=\varphi$ and $D=\psi$. So

(6.1) $\quad \varphi(\Gamma)=C=(-1) W_{3 / 2}^{1 / 2}\left(\frac{1}{2}\right)$

(by equation (5.14))

$$
\begin{aligned}
& =\left[\tau \bar{\tau}\left(1-(\tau /|\tau|)^{2}\right)\right]^{-1} \sum_{\varrho \neq 0} G_{3 / 2}^{1 / 2}\left(\varrho / \tau, \frac{1}{2}\right) \\
& =\left(\tau \bar{\tau}-\tau^{2}\right)^{-1} \sum_{\varrho \neq 0} \wp(\varrho / \tau, \Gamma)
\end{aligned}
$$

(by Lemma 5.1) 
since

$$
G_{3 / 2}^{1 / 2}\left(z, \frac{1}{2}\right)=\sum_{\Omega}\left\{\frac{1}{(z+\Omega)^{2}}-\left\langle\frac{1}{\Omega^{2}}\right\rangle\right\}=\wp(z)
$$

by equation (5.2). Now put $\Gamma=\boldsymbol{b} x$. Since $\varrho / \tau$ has finite order modulo $\boldsymbol{b} x$, it follows from $(6.1)$ that $\varphi(\boldsymbol{b} x)$ is algebraie.

Suppose now that $U(z)$ is a substandard function and that $z$ has order $m$ modulo $b x$. Then $U(z)$ is a polynomial with rational coefficients in $\wp(z), \wp^{\prime}(z), h(z), g_{2}, g_{3}$, and $\varphi$. Each of these is algebraic, hence so is $U(z)$. By Lemma 5.2, $\psi^{n / 2-s_{0}} F_{n}\left(z, s_{0}, \boldsymbol{b} x\right)=U(z)+B$, where $U$ is substandard and $B=0$ unless $s_{0}=1$. If $s_{0}=1$, then (5.9) shows that $B$ is algebraic. Hence $\psi^{n / 2-s_{0}} F_{n}\left(z, s_{0}, \boldsymbol{b} x\right)$ is algebraic. Q.E.D.

We now proceed to prove Theorem 1 . Let $\boldsymbol{a}_{1}, \ldots, \boldsymbol{a}_{h}$ be ideals of $\boldsymbol{S}$, prime to $m$, representing the proper ideal classes $A_{1}, \ldots, A_{h}$ of $\boldsymbol{S}$. The ideal $\boldsymbol{a}_{i}^{-1}$ lies in $A_{i}^{-1}$ and the integral ideals of this class have the form

$$
\boldsymbol{a}=[\alpha] \boldsymbol{a}_{i}^{-1}
$$

with $\alpha$ in $\boldsymbol{a}_{i}$. Then the series $(2.2)$ for $\zeta(s, \lambda)$ ean be witten

$$
\zeta(s, \lambda)=\sum \frac{\lambda\left([\alpha] \boldsymbol{a}_{i}^{-1}\right)}{\mathscr{N}\left([\alpha] \boldsymbol{a}_{i}^{-1}\right)^{s}}=\sum_{i=1}^{\hbar} \frac{\mathscr{N}\left(\boldsymbol{a}_{i}\right)^{s}}{\lambda\left(\boldsymbol{a}_{i}\right)} \sum_{[a] \subseteq \boldsymbol{a}_{i}} \frac{\lambda([\alpha])}{\mathscr{N}([\alpha])^{s}} .
$$

If $e$ is the number of units of $\mathbf{S}$, each [a] has just $e$ generators, and the inner sum can be converted into a sum over numbers. Substitute for $\lambda$ from $(2.1)$ and put $\mathscr{N}([\alpha])=\alpha \bar{\alpha}$. This gives

$$
\zeta(s, \lambda)=e^{-1} \sum_{i=1}^{h} \frac{\mathscr{r}\left(\boldsymbol{a}_{i}\right)^{s}}{\lambda\left(\boldsymbol{a}_{i}\right)} \sum_{\alpha \in \boldsymbol{a}_{i}} \frac{\bar{a}^{n / 2-s}}{a^{n / 2+s}} \chi(\alpha) .
$$

Let $\boldsymbol{b}=\boldsymbol{m a}_{\boldsymbol{i}}$. Let $\beta$ run through a set of representatives of residues of $a_{i}$ modulo $b$, and put $\alpha=\beta+\mu$, where $\mu \epsilon b$. Then $\chi(\alpha)=\chi(\beta)$ and

$$
\zeta(s, \lambda)=e^{-1} \sum_{i=1}^{h} \frac{\mathscr{N}\left(\boldsymbol{a}_{i}\right)^{s}}{\lambda\left(\boldsymbol{a}_{i}\right)} \sum_{\beta \bmod \boldsymbol{b}} \chi(\beta) \sum_{\mu \in \boldsymbol{b}} \frac{(\bar{\beta}+\bar{\mu})^{n / 2-s}}{(\beta+\mu)^{n / 2+s}} .
$$

Multiply both sides by $\left.\pi^{n / 2-s} / x^{n}=\left(\bar{x}^{n / 2-s} / x^{n / 2+8}\right)(\pi / x) \bar{x}\right)^{n / 2-s}$. (This is single valued by the branch convention if we choose some value of $\log (\pi)$.) Then

$$
\begin{aligned}
& \pi^{n / 2-s} \zeta(s, \lambda) / x^{n} \\
& =e^{-1} \sum_{i=1}^{h} \frac{N\left(\boldsymbol{a}_{i}\right)^{s}}{\lambda\left(\boldsymbol{a}_{i}\right)} \sum_{\beta \bmod \boldsymbol{b}} \chi(\beta)(\pi / x \bar{x})^{n / 2-s} \sum_{\mu \in \boldsymbol{b}}^{\mathcal{Y}} \frac{(\bar{\beta} \bar{x}+\bar{\mu} \bar{x})^{n / 2-s}}{(\beta x+\mu x)^{n / 2+s}}
\end{aligned}
$$

The innermost sum is $\mathbb{F}_{n}(\beta x, s, \boldsymbol{b} x)$, so $\zeta(s, \lambda)$ is regular at $s=s_{0}$ by Lemma ๖.2. Put $s=s_{0}, p=\frac{1}{2} n-s_{0}$ and substitute for $\pi / x \bar{x}$ from (4.5). Substitute for $\lambda\left(\boldsymbol{a}_{i}\right)$ from (2.1) and put $\mathscr{N}\left(\boldsymbol{a}_{i}\right)=\left|\hat{\boldsymbol{a}}_{i}\right|^{2}=\hat{\boldsymbol{a}}_{i} \overline{\hat{\boldsymbol{a}}}_{i}$. This gives

(6.2) $\Theta=\pi^{n / 2-s_{0}} \zeta\left(s_{0}, \lambda\right) / x^{n}$

$$
=e^{-1}\left(-\frac{1}{2} \mathscr{r}(\boldsymbol{m}) f \sqrt{\left.|d|\right|^{p}} \sum_{i=1}^{n} \hat{\boldsymbol{a}}_{i}^{n} \chi^{-1}\left(\hat{\boldsymbol{a}}_{i}\right) \sum_{\substack{\beta \in \boldsymbol{\epsilon}_{i} \\ \beta \bmod \boldsymbol{b}}} \chi(\beta) \psi^{2} F_{n}\left(\beta x, s_{0}, \boldsymbol{b} x\right)\right.
$$

where $\boldsymbol{b}=\boldsymbol{m a}_{i}$. Now the $\hat{\boldsymbol{a}}_{i}$ are algebraic, the values of $\chi$ are roots of unity, and $\psi^{p} F_{n}\left(\beta x, s_{0}, \boldsymbol{b} x\right)$ is algebraic by Lemma 6.1 . Hence $\Theta$ is algebraic. This proves Theorem 1 .

\section{References}

[1] B. J. Birch and H. P. F. Swinnerton-Dyer, Notes on elliptic curves, II, J. Reine Ang. Math. 218 (1965), pp. 79-108.

[2] J. W. S. Cassels, A note on the division values of $\wp(u)$, Proc. Camb. Phil. Soc. 45 , part 2, (1948), pp. 167-172.

[3] - Diophantine equations with special reference to elliptic curves, J. London Math. Soc. 41 (1966), pp. 193-291.

[4] M. Deuring, Die Zetafunltion einer algebraischen Kurve vom Geschlechte Eins, I, Nachr. Akad. Wiss. Göttingen (1953), pp. 85-94.

[5] R. Fricke, Die elliptischen Funktionen und ihre Anvendungen, I, B. G. Teubner, Leipzig and Berlin. Page references have been given for this source, and these apply to the edition of 1916.

[6] H. Hasse, Neue Begrundung der komplexen Multiplitation, $I, J$. Reine Ang. Math. 157. (1927), pp. 115-139.

[7] - - II, J. Reine Ang. Math. 165 (1931), pp. 64-88.

[8] E. Hecke, Eine neue Art von Zetafunktion und ihre Beziehungen zur Verteilung der Primzahten, XI, Math. Zeitschr. 6 (1920), pp. 11-51. Reprinted in "Mathematische Werke".

[9] H. P. F. Swinnerton-Dyer, Oaleulation of Tate Products, Private communication.

ROYAL HOLLOWAY COLLWGH

UNIVERSITY OF LONDON 